\title{
ESTÁNDARES E INSTRUMENTOS DE EVALUACIÓN, EN LA PERTINENCIA Y CONFIABILIDAD DE LOS RANKINGS DE UNIVERSIDADES
}

\author{
Elías MEJÍA MEJÍA \\ Universidad Nacional Mayor de San Marcos \\ emejiam@unmsm.edu.pe \\ Edgard DAMIÁN NÚÑEZ \\ Universidad Nacional Mayor de San Marcos \\ edamiann@unmsm.edu.pe \\ Abelardo CAMPANA CONCHA \\ Universidad Nacional Mayor de San Marcos \\ acampanac@unmsm.edu.pe \\ Francis DÍAZ FLORES \\ Universidad Nacional Mayor de San Marcos \\ francis.diaz@unmsm.edu.pe \\ Ofelia SANTOS JIMÉNEZ \\ Universidad Nacional Mayor de San Marcos \\ osantosj@unmsm.edu.pe
}

\section{RESUMEN}

La presente investigación se ha realizado con la finalidad de establecer la influencia que producen, en la pertinencia y la confiabilidad de los Rankings de universidades, los estándares y los instrumentos de evaluación que se aplican para elaborar dichos rankings. La diversidad de rankings, la diversidad de criterios con los que se elaboran y la diversidad de agencias que los elaboran es el mayor indicador que, en este aspecto, no existe uniformidad ni univocidad que propicie la objetividad de estos trabajos, por lo que se ha partido del siguiente problema: ¿Cómo afectan los diversos estándares y los instrumentos de evaluación que aplican las instituciones especializadas, la pertinencia y la confiabilidad de los rankings de universidades que proponen?. Luego de realizar el trabajo de campo, que ha consistido en revisar la literatura especializada, recopilar juicios de expertos, analizar la documentación respectiva y sistematizar la información recopilada, se ha establecido la relación que existe entre los estándares e instrumentos que aplican las distintas instituciones con la pertinencia y confiabilidad de los rankings de universidades que proponen.

\section{PALABRAS CLAVE}

Estándares, instrumentos, calidad de la educación, rankings de universidades.

\section{STANDARDS AND EVALUATION INSTRUMENTS, ON THE RELEVANCE AND RELIABILITY OF THE RANKINGS OF UNIVERSITIES}

\section{ABSTRACT}

This research was conducted in order to establish influence that produced, in the relevance and reliability of the Rankings of universities, standards and evaluation 
instruments used to develop these rankings.

Diversity of rankings, the diversity of criteria that are elaborated and the diversity of agencies that produce them is the greatest indicator that, in this respect, there is no uniformity or uniqueness that is conducive to objectivity and quality, so it has been assumed in the next problem: How does affect the different standards and evaluation instruments that apply specialized institutions, the relevance and the reliability of the rankings of universities proposed?. After carrying out the fieldwork, which has consisted of reviewing specialized literature, collecting expert judgments, analyzing the respective documentation and systematizing the information collected, the relationship between the standards and instruments applied by different institutions with the relevance and reliability of the rankings of universities they propose.

\section{KEY WORDS}

Standards, instruments, quality of education, university rankings.

Recibido: $11 / 03 / 2019$

Aprobado: 06/05/2019

\section{INTRODUCCIÓN}

\footnotetext{
omo resultado del trabajo de campo, hemos analizado los estándares, instrumentos y criterios aplicados para establecer los diferentes rankings de universidades que proponen las instituciones que los elaboran.

Lainvestigación es un estudio comparado entre los estándares einstrumentos con respecto a la pertinencia y confiabilidad de los rankings de universidades que se proponen a nivel internacional.
}

Todo ranking de universidades debe partir de un modelo de evaluación basado en estándares justificados y en la descripción de los instrumentos aplicados para establecer tales rankings de universidades, (Lloyd, 2012). Esta situación no ocurre porque no se disponen de los criterios ni de la respectiva ponderación que permita uniformar los estándares y los instrumentos más pertinentes. Existe diversidad de estándares e instrumentos que hacen dudosa la validez de los criterios para establecer rankings de universidades.

Cuando se analizan tales rankings, notamos la ausencia de estándares consensualmente para las universidades. Unas instituciones trabajan con unos estándares, mientras que otras emplean estándares diferentes. Evaluaciones realizadas con diferentes estándares producen, como consecuencia, diferentes resultados $y$, consecuentemente, los rankings que proponen no resultan pertinentes ni confiables.

En muchos casos se observa que cada institución elabora sus propios estándares en base a la realidad interna de cada institución. Esto no es pertinente, porque si 
se trata de establecer criterios objetivos para plantear rankings de universidades, éstos deben ser realizados con procesos de estandarización adecuados y con instrumentos previamente validados.

\section{OBJETIVOS}

Los objetivos de la presente investigación son los siguientes.

- Identificar los estándares que seaplican para establecer rankings de universidades.

- Analizar los instrumentos aplicados en los procesos de elaboración de los rankings de universidades, de modo tal que éstos resulten pertinentes y confiables.

- Proponer un sistema básico de estándares para evaluar universidades con criterios ponderados y basados en la objetividad.

Como se ha dicho, no existe un sistema consensuado de estándares ni de instrumentos para establecer rankings de universidades, tanto a nivel nacional como internacional. Las instituciones y agencias que elaboran estos rankings emplean diferentes criterios para seleccionar los estándares y emplean diversos instrumentos para elaborar rankings de universidades. En la mayoría de los casos, se basan en encuestas de satisfacción que se aplican en línea. Además, los instrumentos que se aplican asignan diferentes pesos a los estándares, lo que contribuye a incrementar la complejidad de los estudios.

En este sentido, hace falta un estudio basado en la revisión de la literatura con respecto al tema, por lo que proponemos un sistema básico de estándares basados en cuatro dimensiones: La comunidad académica, los procesos académicos, la investigación científica y la internacionalización que, por lo revisado en la literatura, son las dimensiones en las que coinciden con mayor frecuencia, las agencias que elaboran los rankings y, por esta razón, creemos que resulta lo más pertinente.

\section{FUNDAMENTACIÓN Y FORMULACIÓN DE LAS HIPÓTESIS}

En los últimos años, como resultado de una tendencia mundial, ha surgido en las universidades la preocupación por asegurar la calidad de los servicios que brindan. Sin embargo, la apreciación de la calidad no es posible si no se realizan procesos de medición. Sin embargo, como el fenómeno a medir no se muestra plenamente evidente porque la calidad es un concepto valorativo que lleva implícitas muchas connotaciones muy subjetivas, no permite establecer estándares uniformes que podrían aplicarse en diferentes contextos institucionales. Tan grande es el problema que muchas teorías al respecto sostienen que variables de este tipo no se pueden medir, aún más, con la tendencia que cada vez se generaliza del enfoque cualitativo en la investigación científica, muchos especialistas opinan que serían poco confiables y pertinentes los rankings de universidades que se proponen. (Montufar, 2015). 
Por estas consideraciones, los rankings de universidades no resultan pertinentes y son poco confiables. Creemos que una comprensión hermenéutica de los hechos no es suficiente, sino que las universidades deben ser comparadas en base a estándares e instrumentos pertinentes y objetivos.

Es necesario hacer esta comparación para confiar en los rankings propuestos en base a estándares comunes y generalizables. Por estas consideraciones, la presente investigación parte de la siguiente hipótesis: La diversidad de estándares y los instrumentos de evaluación que aplican las instituciones especializadas, son factores que afectan la pertinencia y confiabilidad de los rankings de universidades que proponen.

\section{MÉTODOS}

La investigación se ha realizado mediante el método bibliográfico que ha permitido revisar la información especializada que da cuenta de la diversidad de estándares e instrumentos, así como de distintos criterios y agencias internacionales que elaboran estándares. Como consecuencia de este análisis, hemos adoptado la conclusión que si existe una gran diversidad de estándares e instrumentos, los rankings que se elaboran, como resultado de la aplicación de estos estándares e instrumentos, no son necesariamente pertinentes ni confiables. Es así como unas universidades, según ciertos estándares, se ubican en los lugares más elevados de la escala, mientras que las mismas universidades, según otros criterios, se ubican en lugares más bajos de la escala.

La revisión de la literatura respectiva y la opinión de expertos, ha permitido identificar los diversos estándares, para luego analizar y sistematizar la información así obtenida. (De la Orden, Arturo 2007).

Se ha elaborado un instrumento, con una estructura que pretende ser integral, en base a los estándares analizados. Finalmente hemos procedido a analizar los datos así obtenidos para decidir cuan pertinentes y confiables son los rankings de universitarios propuestos en base a los estándares e instrumentos que aplican las instituciones que los elaboran.

Los antecedentes sobre los rankings de universidades son muchos y cada vez es más intensa la preocupación por los criterios que se emplean para establecer rankings de universidades.

En el contexto internacional, existen diferentes rankings de universidades elaboradas según diferentes criterios. Algunas universidades ocupan un lugar más alto en los rankings en base a estándares referidos a locales e infraestructura que poseen. Otros aparecen en los rankings en base a la producción científica, mientras que otras aparecen en los rankings en base al impacto de sus páginas Web.

Sin embargo, el análisis de tales rankings es poco confiable pues no se han validado los estándares según los cuales se ha establecido dicho ranking. Y además porque 
el problema de la calidad de la educación es tan compleja y multidimensional, (Roa Varelo, 2003).

\section{RESULTADOS}

Las principales agencias que elaboran rankings de universidades a nivel internacional son las siguientes:

\subsection{ACADEMIC RANKING WORLD UNIVERSITIES, (ARWU).}

Según Krüger, (2010), es el más antiguo y posiblemente con este ranking se ha iniciado toda esta tendencia de jerarquizar universidades. Apareció por primera vez el año 2003, y fue publicado por el Instituto de Educación Superior de la Universidad de Jiao Tong de Shanghai. En base a una fuerte actitud competitiva, las autoridades de esta Universidad deseaban conocer la calidad y la efectividad de las universidades chinas de investigación y compararlas con otras universidades del mundo. Este ranking, posiblemente por razones de difusión, se ha convertido en el referente de todos los sistemas de elaboración de rankings de universidades y casi todos los demás rankings emplean una metodología similar a este sistema.

Lo más característico es, contrariamente a lo que podría suponerse, que el ARWU no evalúa todas las universidades, sino solo un conjunto reducido constituido por aquellas universidades que, según sus estándares y sus criterios, han pasado por un primer filtro. Los estándares que aplican para filtrar las universidades son muy altos, tales como si las universidades que se van a evaluar tienen ganadores de Premios Nobeles, o ganadores de la Medalla Fields, en el caso de distinciones para especialistas en matemática, o investigadores con un alto índice de citaciones.

Otro estándar es si la mayoría de universidades de cada país tienen una cierta cantidad de artículos indexados en el Sciencie Citation Index-Expanded (SCIE) y el Social Citation Index (SSCI). Porque estos indexadores son parte de la Web of Science que, a su vez, es propiedad de la Thomson Reuter que, dicho sea de paso, es una empresa privada.

El ARWU trabaja con aproximadamente 2000 universidades que habrían pasado estos filtros. De este total, según Krüger, (2010), solo evalúan a 1200 universidades y, en el ranking, aparecen sólo las primeras 500 universidades que, según sus criterios, lo merecen.

El ARWU clasifica las universidades en los siguientes cinco campos del conocimiento:

1. Ciencias naturales y matemáticas,

2. Ingeniería, tecnología y ciencias de la computación.

3. Ciencias de la vida y la agricultura.

4. Medicina y farmacia $y$,

5. Ciencias sociales. 
Es curioso notar que el ARWU no considera las Artes ni las Humanidades porque asumen que no existen estándares objetivos ni internacionalmente comparables en estas áreas.

El orden se establece en base al porcentaje de artículos publicados entre los mejores y los gastos de investigación.

El ranking del 2008, según Krüger, (2010), se elaboró en base a los siguientes indicadores:

1. Calidad de la educación, entendida básicamente con los resultados del proceso, es decir, los egresados de la Universidad que se evalúa que hayan ganado algún Premio Nobel en Física, Química, Medicina o Economía, o la Medalla Fields, para la excelencia en Matemática.

2. Calidad de las facultades, en función del número de profesores que, cuando trabajaban en sus facultades, ganaron Premios Nobles o Medallas Fields.

3. Resultados de investigaciones, estimados en base al número de artículos publicados en ciencias naturales. Los artículos se clasifican por campos del conocimiento que los establece el Clasification of Journal Categories. Esta base de datos reconoce cinco campos del conocimiento: Medicina. Física, Química, Biología y Economía.

4. Rendimiento per cápita. Este indicador se establece dividiendo el número de publicaciones entre el número de profesores que trabajan en la Universidad.

\subsection{WORLD UNIVERSITY RANKING DEL TIMES HIGHER EDUCATION}

Conocido como el THES-Ranking, evalúa alrededor de 600 universidades en el mundo y publica una clasificación de las 400 mejores. Se elabora en base a seis indicadores y en las siguientes áreas del conocimiento: Artes y Humanidades, Ingeniería y Tecnología, Ciencias de la vida y Biomedicina, Ciencias Naturales, y Ciencias Sociales.

El fundamento en que se basa el THES es el reconocimiento de las universidades como organizaciones multifacéticas y que cuando se trata de la comparación de sus éxitos, pugnan por convertirse en las mejores o de clase mundial. El THES fomenta la competencia, por tener mejores profesores, mejores estudiantes, lograr contratos de investigación más lucrativos, y obtener más fondos gubernamentales. En este sentido, la base de datos denominada World Class University, adquiere mayor importancia para los gobiernos, los empleadores, los inversionistas, los estudiantes y las mismas universidades

El World University Ranking establece cuatro criterios de calidad:

1. Calidad de la investigación,

2. Calidad de la enseñanza,

3. Empleabilidad de los graduados y

4. Imagen internacional.

La metodología de estudio del World University Ranking se realiza mediante encuestas en línea sobre las siguientes dimensiones: 
a. Perspectiva académica, considerada la más importante y es ponderada con el $40 \%$. El estudio se hace mediante preguntas en líneas a los académicos, suscriptores de World Scientific y del International Book Information Services, para que identifiquen las universidades que ellos consideran sobresalientes en el campo del conocimiento. Los encuestados no pueden mencionar su propia universidad y trabajan con más de 600,000 direcciones electrónicas. Las respuestas se ponderan por áreas geográficas entre las siguientes regiones: América; Europa, Medio Oriente y África y Asia Pacífico.

b. Expectativas de los empleadores, para establecer la empleabilidad de los egresados, preguntándoles si contratan graduados de las universidades que ellos consideran preparan mejor para los puestos de trabajo que disponen.

c. Calidad de la enseñanza, que se reconoce como el indicador más aceptable. Mide la calidad de la enseñanza en base a la tasa de estudiantes por universidad, lo que significa que cuanto la tasa es menor, la calidad se incrementa.

d. Prestigio científico, que es el resultado de la combinación de datos sobre la productividad y la calidad científica.

e. Internacionalización, en base a estudiantes y profesores de distintos países que es estudian o trabajan en la universidad evaluada.

\subsection{NEW GLOBAL UNIVERSITY RANKING (RATER-RANKING)}

Los elabora el Instituto ruso Rating of Educational Resources (Rat-ER) y se propone los mismos objetivos del $A R W U$, pero en función de Rusia. Este ranking pretende elaborar una clasificación alternativa al $A R W U$ y al THES, en los que las universidades de habla inglesa tienen mayor ventaja.

Emplea indicadores numéricos comparables y tiene en cuenta, a diferencia del $A R W U$, la investigación científica y las actividades pedagógicas porque el estado ruso pretende una educación de calidad basada en los estándares establecidos por el gobierno y tenga en cuenta los requerimientos del mercado laboral.

Este ranking trabaja con las siguientes dimensiones:

1. Aspectos académicos de la universidad.

2. Calidad de la investigación científica que desarrolla.

3. Prestigio científico de la universidad en la comunidad internacional.

4. Satisfacción de los recursos requeridos.

5. Actividades relevantes de sus graduados.

6. Proceso de internacionalización de la universidad.

\subsection{SCIMAGO INSTITUTIONAL RANKING WORLD REPORT}

Este ranking es accesible por su página Web. Lo elaboran investigadores de universidades españolas y portuguesas y del Instituto de Políticas y Bienes Públicos del CSIC español. El Scimago Institutional Ranking, más conocido como SIR, evalúa la calidad de la investigación científica de más de 2,000 universidades de 84 países de los cinco continentes, a partir de los artículos científicos que publican y las citas que merecen y que aparecen en Scopus de Elsevier. (ANECA, 2015). 
Su metodología es bibliométrica y utiliza exclusivamente la base de datos de Scopus, según los siguientes componentes:

1. Outpunt, que se calcula por el número de documentos publicados en revistas científicas de la base de datos. Este indicador está influido por el tamaño de la institución y por el perfil del investigador.

2. Citas de los artículos publicados, que nuestra el impacto de la producción científica de la universidad. Este indicador expresa el promedio de citas de los documentos publicados en un determinado periodo.

3. Colaboración internacional, que corresponde a la intensidad de la colaboración con otras universidades extranjeras para la publicación de sus producciones. El componente recibe más puntaje en función del número de investigadores extranjeros que intervienen.

4. Puntuación de citas normalizado por campo del conocimiento que expresa la relación entre el promedio del impacto científico de una institución y el promedio de su impacto internacional

\subsection{PERFORMANCERANKING OF SCIENTIFICPAPERSOFWORLDUNIVERSITIES}

Este ranking lo publica el Hihger Education Evaluation and Acreditation Council of Taiwan y evalúa alrededor de 500 universidades de clase mundial, en base a la investigación científica que realizan. Sobre esta base trata de establecer estándares más objetivos y no en base a los premios internacionales o encuestas de opinión sobre la reputación científica de las universidades. Es una clasificación bibliométrica de la calidad de sus investigadores.

Su metodología emplea los datos del Thomson Reuters, como el Essential Science Indicators, o la Web of Science que registra el índice de citaciones de ciencias. También analiza las mismas publicaciones científicas, pero excluye las citas en Artes y Humanidades, debido a que en estas áreas los investigadores publican la mayoría de sus trabajos en su propio idioma mientras que los investigadores del campo de las ciencias lo hacen en inglés.

Emplea los siguientes componentes:

1. Productividad en investigación, en función del número de artículos publicados en los últimos 11 años.

2. Impacto de la investigación, en función del número de citas en los últimos 11 años.

3. Excelencia de los investigadores, en función al Índice $\mathrm{H}$ de los investigadores en los últimos años y el número de investigaciones altamente citadas.

\subsection{RANKING IBEROAMERICANO DE UNIVERSIDADES POR CALIDAD INVESTIGADORA}

Constituye parte del proyecto I+D "Atlas de la Ciencia" que elabora Scimago. Divide a las universidades en dos regiones geográficas: los países iberoamericanos y los países latinoamericanos. En este proyecto aparecen Argentina, Brasil, Chile, Colombia, Cuba, España, México, Perú, Portugal y Venezuela. (ANECA, 2015). 
Este también es un ranking bibliométrico que se basa exclusivamente en el análisis de las publicaciones científicas a partir de la base de datos de la Thomson Scientific. Como se deduce, este ranking sólo tiene en cuenta la producción científica.

Su metodología evalúa solamente las universidades que han producido en el periodo evaluado más de 100 artículos y utiliza los siguientes componentes:

1. Producción científica total.

2. Producción citable o producción primaria.

3. Producción ponderada o potencial investigador.

4. Impacto institucional ponderado.

5. Colaboración internacional.

\subsection{RANKING WEB DE UNIVERSIDADES EN EL MUNDO}

Lo elabora el Laboratorio de Cybermetría del CSIC de España. Analiza investigaciones que aparecen en la Web, pero no se centra en los resultados de las investigaciones sino promueve, entre los profesores universitarios, publicar en la Web como una alternativa de reducir la brecha digital.

La unidad de análisis de la que parte es el prestigio institucional, por lo que solo aquellas universidades, con una presencia preponderante en la Web, son tomadas en consideración. El componente de más peso es el impacto que produce la universidad en la Web que además se combina con el número de enlaces externos entrantes y con el número de páginas Web de un dominio. Además aplica los siguientes componentes:

1. Tamaño de la Universidad.

2. Visibilidad en la Web.

3. Abundancia de información.

4. Personal académico.

\subsection{TIMES HIGHER EDUCATION WORLD UNIVERSITY RANKINGS}

Es una agencia norteamericana que la dirige Phil Baty. Los rankings generalmente se usan en el mundo para establecer políticas públicas para la educación superior. Sin embargo, el mismo Baty, (Lloyd, 2012), en una serie de artículos critica sus propios rankings y afirma que éstos deben usarse como guía o referencia para apreciar la calidad del servicio que brindan las universidades y no como resultados imparciales ni mucho menos definitivo la calidad del servicio que brindan las universidades.

\section{DISCUSIÓN}

\section{LOS RANKINGS DE UNIVERSIDADES}

Los rankings se elaboran, en la mayoría de los casos, en base a encuestas de satisfacción de los supuestos clientes. Se debe tener en cuenta que una encuesta 
es un conjunto de opiniones acerca de lo que se desea saber y por esto es poco confiable establecer la calidad del servicio que brinda una universidad en base a opiniones. Estas opiniones son, como se ha dicho, de satisfacción, es decir los clientes opinan acerca de si se encuentran satisfechos o no con el servicio que han recibido.

Otras encuestas están dirigidas a los empleadores de los graduados de las universidades. Ellos opinan acerca del desempeño de los egresados que trabajan para ellos. Pero también es dudosa la posibilidad de medir la calidad de una universidad por el desempeño de algunos de sus egresados o la opinión de algunos de sus empleadores.

No siempre los egresados son los indicadores más fiables para establecer la calidad de la universidad que lo formó. Es posible la aparición de ciertos sesgos pues no todos los egresados tienen el mismo desempeño profesional. Eso depende, muchas veces, del esfuerzo y características personales y motivaciones de los profesionales que deben enfrentar en el mercado laboral.

Según Lloyd, (2012), la gran tendencia de los últimos años de establecer rankings de universidades, se inició en 2003 con la publicación del primer ranking internacional, el aludido Academic World Ranking de Universidades, publicado por la Universidad de Jiao Tong de Shanghai. Esta publicación se hizo con fines comparativos pues los académicos de Shanghai querían saber cómo se ubicaban en lo referido a la producción científica, comparándose con las universidades de "clase mundial". En este ranking no tiene en cuenta la calidad de la docencia ni el impacto social que produce.

Los problemas técnicos referidos a las publicaciones son muchos y los Premios Nobeles no se ganan de inmediato, sino que los científicos reciben el reconocimiento por sus investigaciones muchos años después que han publicado sus investigaciones y cuando han dejado de trabajar en la universidad, como es el caso de Einstein, a quien concedieron el Premio Nobel, no por la teoría de la relatividad, sino por un artículo que publicó con casi quince años de anterioridad.

Estos estándares aparentemente se pueden medir muy objetivamente y en función de ellos se pueden establecer los rankings. Sin embargo, cuál sería el estándar mínimo de Premios Nobel para definir a una universidad como de clase mundial?

Por eso han aparecido otros rankings como el de la Universidad de Leiden (Lloyd, 2012), y el Scimago Instituctional Ranking (SIR) que miden exclusivamente la producción científica de las universidades.

Posteriormente, ha aparecido la británica Quacquarelli Simons (QS) que añade otros rubros, como el número de estudiantes por profesor. Sin embargo los resultados siempre son subjetivos y sesgados acerca de la calidad de la universidad.

Otro sesgo importante es que las agencias norteamericanas, establecen comparaciones con los estándares de Harvard. Es imposible que las universidades latinoamericanas alcancen los estándares de Harvard, por eso es improcedente 
compararlas con esta universidad. De ahí surge la necesidad de establecer ciertos estándares más objetivos que realmente reflejen la calidad del servicio.

Lloyd, (2012), refiere que en el Encuentro de las Universidades Latinoamericanas ante los Rankings Internacionales: impactos, alcances y límites, realizado en la UNAM en 2010, más de 60 rectores de la región emitieron una Declaración Final en la que expresaban su inconformidad con el uso de los rankings para la formulación de políticas públicas. Lloyd cuestiona el fuerte y casi exclusivo estándar de producción científica que favorece a un solo modelo de universidad, la universidad anglosajona de investigación, de la cual Harvard es el mejor ejemplo. Immanol Ordorika, citado por Lloyd, (2012), uno de los organizadores del certamen, afirmó con respecto es estos estándares, que "estos fungen como harvadómetros, qué tanto te pareces a Harvard"

Es notoria la falta de transparencia de las agencias que elaboran los rankings. Las agencias no publican sus estándares, ni sus instrumentos, ni tampoco sus metodologías, por lo que son poco confiables y la alta fluctuación de las universidades, de un año para otro, es un claro indicador que los rankings no son objetivos, sino sesgados y subjetivos.

EI QS se considera el más comercial de los rankings porque busca colocarse en el mercado de la educación superior a través de nuevos productos. Por este motivo publica el Ranking Latinoamericano de Universidades. Pero además QS ofrece consultorías a las universidades que buscan mejorar su ubicación en los rankings, convirtiéndose de este modo en juez y parte, lo que a todas luces es contradictorio con la imparcialidad con la que debe actuar una agencia evaluadora de universidades. Sin embargo QS se ha posicionado como la agencia de mayor credibilidad en la región.

En una de sus últimas publicaciones, considera a 65 universidades del Brasil entre las 200 primera en América Latina. Y la mayoría de las universidades brasileñas son privadas, lo que indica el sesgo privatizador de este ranking.

\section{LOS ESTÁNDARES DE CALIDAD}

Por otra parte, el concepto de estándar, también es muy controversial. Nosotros entendemos por estándar la condición mínima requerida para asignar un valor o característica a algo. Así, para establecer si una universidad posee calidad, debe satisfacer ciertos estándares mínimos. (Torranzos, 2014).

Algunos autores no asumen este mismo criterio y emplean el término como sinónimo de los instrumentos que aplican. Los estándares varían en función de la institución que realiza la evaluación. No hemos hallado ningún instrumento común que se aplique a la evaluación de universidades. (González, Ignacio y López, Isabel, 2010).

Ante esta situación, proponemos un sistema organizado de estándares que es resultado del estudio comparado que hemos realizado y que obedece una estructura que la consideramos pertinente debido a que se incluye los componentes de evaluación más comunes que se aplican, pero hemos añadido la respectiva ponderación, lo que no sucede con los estudios que hemos revisado. Así, con 
este criterio de ponderación podemos establecer la influencia de mayor o menor o repercusión en la calidad del servicio que brindan las universidades de ciertos componentes de modo tal que si se deseara mejorar la calidad, se podrá actuar en forma focalizada en el componente evaluado como deficitario y, para mantener la calidad, se deberá fortalecer los componentes evaluados satisfactoriamente.

Estos componentes se justifican porque entendemos que las universidades deben definirse por lo que realmente son, es decir, por que realizan formación profesional y porque realizan actividades de investigación. El sesgo que hemos hallado es que la mayoría de las agencias que elaboran los rankings lo hacen solo en base a las actividades de investigación de las universidades,

Sin embargo, las mismas agencias que elaboran los rankings asumen actitudes autocríticas con respecto a que la calidad de la universidad no se puede estimar solamente en base a indicadores cuantitativos y reconocen que los rankings no son objetivos.

Por otra parte, medir la calidad de universidades de todo el mundo, es prácticamente imposible a causa de la gran diferencia que existe entre las universidades y los sistemas educativos propios de cada país. Otros problemas son metodológicos como la ponderación de los estándares e indicadores y el rendimiento per cápita de los profesores de la universidad.

En los países altamente desarrollados, las universidades se especializan, en universidades de investigación o productoras de conocimientos mientras que otras universidades forman profesionales. La realidad latinoamericana no permite estas distinciones y la mayoría de las universidades latinoamericanas cumplen las dos tareas: la formación profesional y la investigación.

Además, en algunos países latinoamericanos, especialmente en el Perú, las universidades cumplen una tercera función que es la llamada responsabilidad social. Pero es el caso que por esta función que cumplen, ninguna agencia evalúa su calidad.

\section{PROPUESTA DE ESTÁNDARES MÍNIMOS PARA UNIVERSIDADES NACIONALES}

\begin{tabular}{|c|c|c|c|}
\hline DIMENSIONES & PESO & FACTORES & PESO \\
\hline \multirow{3}{*}{ CONMUNIDAD ACADÉMICA } & \multirow{3}{*}{30} & Docentes & 15 \\
\hline & & Estudiantes & 7 \\
\hline & & Egresados & 8 \\
\hline \multirow{3}{*}{ PROCESOS ACADÉMICOS } & \multirow{3}{*}{25} & Currículo & 10 \\
\hline & & Interacción didáctica & 8 \\
\hline & & Métodos y materiales & 7 \\
\hline \multirow{3}{*}{ INVESTIGACIÓN } & \multirow{3}{*}{30} & Políticas de investigación & 5 \\
\hline & & Publicaciones científicas & 20 \\
\hline & & Investigaciones en curso & 5 \\
\hline \multirow{2}{*}{ INTERNACIONALIZACIÓN } & \multirow{2}{*}{15} & Presencia en la comunidad internacional & 8 \\
\hline & & Colaboración internacional & 7 \\
\hline TOTALES & 100 & & 100 \\
\hline
\end{tabular}




\section{ESTÁNDADES DE LA DIMENSIÓN COMUNIDAD ACADÉMICA}

\subsection{DOCENTES}

Los estándares poseen sus respectivos criterios según los cuales se decide si cumplen o no el estándar. Por esta razón presentamos a continuación el estándar y su respectivo criterio de evaluación.

\begin{tabular}{|l|l|l|}
\hline \multicolumn{1}{|c|}{ Código } & \multicolumn{1}{|c|}{ Estándar } & \multicolumn{1}{c|}{ Criterio } \\
\hline 1.1.1. & Posesión de un título profesional de tercer nivel. & $100 \%$ \\
\hline 1.1 .2$. & Posesión de un grado académico de magíster & $100 \%$ \\
\hline 1.1 .3$. & Posesión de un grado académico de doctor & $90 \%$ \\
\hline 1.1 .4$. & Movilidad en la carrera profesional & Existencia del Plan. \\
\hline 1.1 .5$. & Participación en actividades de capacitación permanente & Existencia del Plan. \\
\hline 1.1.6. & Acceso a incentivos para la capacitación. & $\begin{array}{l}\text { Existencia del } \\
\text { Sistema. }\end{array}$ \\
\hline 1.1.7. & Publicaciones científicas. & $80 \%$ \\
\hline 1.1 .8$. & Presencia nacional e internacional del docente. & $50 \%$ \\
\hline 1.1 .9$. & Premios nacionales o internacionales & $50 \%$ \\
\hline
\end{tabular}

\subsection{ESTUDIANTES}

\begin{tabular}{|l|l|l|}
\hline \multicolumn{1}{|c|}{ Código } & \multicolumn{1}{|c|}{ Estándar } & \multicolumn{1}{c|}{ Criterio } \\
\hline 1.2.1. & Ingresaron por el sistema regulado de admisión. & $100 \%$. \\
\hline 1.2.2. & Culminación de los estudios en los tiempos previstos. & $\begin{array}{l}90 \% \text { culmina } \\
\text { en los tiempos } \\
\text { previstos. }\end{array}$ \\
\hline 1.2.3. & Participación en el sistema regulado de movilidad estudiantil & $\begin{array}{l}\text { Existencia del } \\
\text { Sistema. }\end{array}$ \\
\hline
\end{tabular}

\subsection{EGRESADOS}

\begin{tabular}{|l|l|l|}
\hline \multicolumn{1}{|c|}{ Código } & \multicolumn{1}{|c|}{ Estándar } & \multicolumn{1}{c|}{ Criterio } \\
\hline 1.3.1. & Sistema de seguimiento de sus egresados. & Existencia del sistema. \\
\hline 1.3.2. & Participación en el gobierno de la universidad. & $\begin{array}{l}\text { Se cumple lo } \\
\text { establecido en la Ley. }\end{array}$ \\
\hline 1.3.3. & Empleabilidad & $80 \%$ por cohorte. \\
\hline
\end{tabular}

\section{ESTÁNDADES DE LA DIMENSIÓN PROCESOS ACADÉMICOS}

\subsection{CURRÍCULO}

\begin{tabular}{|l|l|l|}
\hline Código & \multicolumn{1}{|c|}{ Estándar } & \multicolumn{1}{|c|}{ Criterio } \\
\hline 2.1.1. & $\begin{array}{l}\text { Las carreras que brinda la Universidad se desarrollan } \\
\text { según un currículo elaborado metodológicamente, } \\
\text { que posee una estructura pertinente y ha sido } \\
\text { aprobado por las instancias correspondiente. }\end{array}$ & $\begin{array}{l}\text { El 100\% de las carreras } \\
\text { poseen un currículo. }\end{array}$ \\
\hline 2.1.2. & Evaluación periódica del currículo. & $\begin{array}{l}\text { Existencia del sistema de } \\
\text { evaluación del currículo. }\end{array}$ \\
\hline
\end{tabular}




\begin{tabular}{|l|l|l|}
\hline 2.1.3. & $\begin{array}{l}\text { Rediseño periódico del currículo como } \\
\text { consecuencia de la evaluación. }\end{array}$ & $\begin{array}{l}\text { Documentos de rediseño } \\
\text { periódico del currículo. }\end{array}$ \\
\hline
\end{tabular}

\subsection{INTERACCIÓN DIDÁCTICA}

\begin{tabular}{|l|l|l|}
\hline Código & \multicolumn{1}{|c|}{ Estándar } & \multicolumn{1}{|c|}{ Criterio } \\
\hline 2.2.1. & $\begin{array}{l}\text { Sistema regulado de los procesos de enseñanza y } \\
\text { de aprendizaje. }\end{array}$ & Existencia del sistema. \\
\hline 2.2.2. & $\begin{array}{l}\text { Desarrollo de los procesos académicos según el } \\
\text { cronograma establecido. }\end{array}$ & $\begin{array}{l}\text { Cronograma académico de } \\
\text { acuerdo a Ley. }\end{array}$ \\
\hline 2.2.3. & $\begin{array}{l}\text { Supervisión de la calidad de los procesos } \\
\text { académicos. }\end{array}$ & $\begin{array}{l}\text { Existencia del sistema de } \\
\text { supervisión. }\end{array}$ \\
\hline
\end{tabular}

\subsection{MÉTODOS Y MATERIALES}

\begin{tabular}{|l|l|l|}
\hline \multicolumn{1}{|c|}{ Código } & \multicolumn{1}{|c|}{ Estándar } & \multicolumn{1}{|c|}{ Criterio } \\
\hline 2.3.1. & Sistema de innovación de métodos didácticos. & Existencia del sistema. \\
\hline 2.3.2. & $\begin{array}{l}\text { Sistema de capacitación docente para realizar } \\
\text { innovaciones metodológicas. }\end{array}$ & $\begin{array}{l}\text { Se cumple lo establecido } \\
\text { en la Ley. }\end{array}$ \\
\hline 2.3.3. & $\begin{array}{l}\text { Sistema regulado de aplicación de métodos } \\
\text { didácticos. }\end{array}$ & Existencia del sistema. \\
\hline 2.3.4. & $\begin{array}{l}\text { Satisfacción del requerimiento de métodos } \\
\text { didácticos. }\end{array}$ & Satisfacción al 100\%. \\
\hline
\end{tabular}

\section{ESTÁNDARES DE LA DIMENSIÓN INVESTIGACIÓN CIENTÍFICA}

\subsection{POLÍTICAS DE INVESTIGACIÓN}

\begin{tabular}{|l|l|l|}
\hline Código & \multicolumn{1}{|c|}{ Estándar } & \multicolumn{1}{|c|}{ Criterio } \\
\hline 3.1.1. & Líneas y prioridades de investigación. & $\begin{array}{l}\text { Existencia de documentos oficiales } \\
\text { que definen líneas y prioridades de } \\
\text { investigación. }\end{array}$ \\
\hline 3.1.2. & $\begin{array}{l}\text { Correspondencia de las investigaciones } \\
\text { conlaslíneasyprioridades deinvestigación. }\end{array}$ & $\begin{array}{l}\text { Sistema regulado para asegurar la } \\
\text { correspondencia. }\end{array}$ \\
\hline 3.1.3. & $\begin{array}{l}\text { Organización de los investigadores en } \\
\text { Grupos de Investigación. }\end{array}$ & $\begin{array}{l}\text { Documentos de reconocimiento de } \\
\text { los Grupos de Investigación. }\end{array}$ \\
\hline 3.1.4. & Financiamiento de la investigación & Satisfacción al $100 \%$. \\
\hline
\end{tabular}

\subsection{PUBLICACIONES CIENTÍFICAS}

\begin{tabular}{|l|l|l|}
\hline Código & \multicolumn{1}{|c|}{ Estándar } & \multicolumn{1}{|c|}{ Criterio } \\
\hline 3.2.1. & La universidad publica revistas indexadas & $\begin{array}{l}\text { Cada Facultad tiene por } \\
\text { lo menos una revista } \\
\text { indexada. }\end{array}$ \\
\hline 3.2.2. & $\begin{array}{l}\text { Los Grupos de Investigación publican sus artículos } \\
\text { científicos. }\end{array}$ & $\begin{array}{l}\text { Un artículo científico anual } \\
\text { por Grupo de Investigación. }\end{array}$ \\
\hline 3.2.3. & $\begin{array}{l}\text { Sistema regulado de evaluación de las } \\
\text { investigaciones publicadas. }\end{array}$ & $\begin{array}{l}\text { Existencia del sistema de } \\
\text { evaluación. }\end{array}$ \\
\hline
\end{tabular}




\subsection{INVESTIGACIONES EN CURSO}

\begin{tabular}{|l|l|l|}
\hline Código & \multicolumn{1}{|c|}{ Estándar } & \multicolumn{1}{|c|}{ Criterio } \\
\hline 3.3.1. & $\begin{array}{l}\text { Investigaciones que se encuentran en en curso } \\
\text { en cada Facultad. }\end{array}$ & $\begin{array}{l}\text { Proyectos en ejecución, por } \\
\text { cada Facultad. }\end{array}$ \\
\hline 3.3.2. & $\begin{array}{l}\text { Las investigaciones en curso han sido aprobadas } \\
\text { previamente. }\end{array}$ & $\begin{array}{l}\text { Documentos de aprobación } \\
\text { de los proyectos de } \\
\text { investigación. }\end{array}$ \\
\hline 3.3.3. & $\begin{array}{l}\text { Seguimiento y monitoreo de las investigaciones } \\
\text { en curso. }\end{array}$ & $\begin{array}{l}\text { Existe un sistema de } \\
\text { monitoreo y supervisión. }\end{array}$ \\
\hline
\end{tabular}

\section{ESTÁNDARES DE LA DIMENSIÓN INTERNACIONALIZACIÓN}

\subsection{PRESENCIA EN LA COMUNIDAD INTERNACIONAL}

\begin{tabular}{|l|l|l|}
\hline Código & \multicolumn{1}{|c|}{ Estándar } & \multicolumn{1}{|c|}{ Criterio } \\
\hline 4.1.1. & $\begin{array}{l}\text { Plan de internacionalización de la Universidad a } \\
\text { corto, mediano y largo plazo. }\end{array}$ & $\begin{array}{l}\text { Existencia de documentos } \\
\text { oficiales que definen } \\
\text { líneas y prioridades de } \\
\text { investigación. }\end{array}$ \\
\hline 4.1.2. & $\begin{array}{l}\text { La universidad aparece en los rankings } \\
\text { internacionales que se publican. }\end{array}$ & $\begin{array}{l}\text { Sistema regulado } \\
\text { para asegurar la } \\
\text { correspondencia. }\end{array}$ \\
\hline 4.1.3. & $\begin{array}{l}\text { La Universidad realiza actividades conjuntas con } \\
\text { otras universidades del extranjero. }\end{array}$ & $\begin{array}{l}\text { Convenios de cooperación } \\
\text { internacional. }\end{array}$ \\
\hline
\end{tabular}

\subsection{COLABORACIÓN INTERNACIONAL}

\begin{tabular}{|c|l|l|}
\hline Código & \multicolumn{1}{|c|}{ Estándar } & \multicolumn{1}{|c|}{ Criterio } \\
\hline 4.2.1. & $\begin{array}{l}\text { Profesores extranjeros que trabajan en la } \\
\text { Universidad. }\end{array}$ & $\begin{array}{l}\text { 5\% de profesores } \\
\text { extranjeros en la } \\
\text { Universidad. }\end{array}$ \\
\hline 4.2.2. & $\begin{array}{l}\text { Profesores extranjeros que participan en proyectos } \\
\text { de investigación. }\end{array}$ & $\begin{array}{l}\text { Sistema regulado } \\
\text { para asegurar la } \\
\text { correspondencia. }\end{array}$ \\
\hline 4.2.3. & $\begin{array}{l}\text { Movilidad de los docentes en universidades } \\
\text { extranjeras. }\end{array}$ & $\begin{array}{l}\text { Convenios de cooperación } \\
\text { internacional. }\end{array}$ \\
\hline
\end{tabular}

\section{CONCLUSIONES}

Luego del proceso de investigación hemos arribado a las siguientes conclusiones:

1. Las instituciones que elaboran rankings de universidades, a nivel internacional son, casi en su totalidad, empresas privadas.

2. La revisión de la literatura ha permitido comprobar que existe un gran número de agencias que elaboran rankings universitarios y, cada una de ellas, lo hace con sus propios estándares e instrumentos. 
3. Los estándares que cada agencia emplea, han sido adoptados a partir de diversos criterios. Así unas agencias empleas unos criterios, como los Premios Nobeles ganados por sus egresados y otras empleas criterios distintos, como la publicación de artículos científicos.

4. Los instrumentos de evaluación de universidades que aplican estas agencias para la elaboración de los rankings, son diversos y no es posible la comparación objetiva entre ellos.

5. Los estándares e instrumentos que aplican las agencias no son pertinentes para elaborar rankings o escalas que realmente establezcan la jerarquía en la que se ubican las universidades. Al ser todos distintos, los cada uno de los rankings, tiene un valor y aplicación diferente con respecto a otro.

6. Están surgiendo opiniones, desde el interior de las universidades, orientadas a cuestionar la pertinencia de los rankings, lo que los hace poco confiables para adoptar, en base a ellos, políticas de mejoramiento de la calidad de la educación superior.

\section{REFERENCIAS BIBLIOGRÁFICAS}

ANECA. (2015). Criterios y directrices para el aseguramiento de la calidad en el Espacio Europeo de Educación Superior (ESG). Recuperado de: https://www. creativecommon.org

De la Orden Hoz, A. (2007). Desarrolloy validación de un modelo de calidad universitaria como base para su evaluación. Recuperado de: https;//www.ojs.uv.es

González, I. y López, I. (2010). Validación y propuesta de un modelo de indicadores de evaluación de la calidad en la universidad. Recuperado de: https://www.rieoei. org

Krüger, K. (2010). Rankings mundiales de universidades: Objetivos y calidad. Recuperado de: https://www.academia.edu

Lloyd, M. (2012). La mea culpa de los ranqueadores. Seminario de Educación Superior de la UNAM.

Montufar, C. (2015). ¿Es el "World University Ranking" un instrumento de clasificación académica válido para la política pública en América Latina? Recuperado de: https://www.internalmedicine.conferences.com

Roa, A. (2003). Hacia un modelo de aseguramiento de la calidad en la educación superior en Colombia: Estándares básicos y acreditación de excelencia. Recuperado de: https://www.cna.gov.co

Torranzos, L. (2014). En la búsqueda de estándares de calidad. Recuperado de: https: //www. docplayer.es 